\title{
Conhecimento e adesão ao aleitamento materno
}

\section{Knowledge and accession to maternal breastfeeding}

Conocimiento y adhesión a la lactancia

\author{
Karla de Toledo Candido Muller ${ }^{1}$ \\ Suellen Zama Barbosa² \\ Débora Cardozo Bonfim Carbone ${ }^{3}$ \\ Durval Batista Palhares ${ }^{4}$
}

${ }^{1}$ Fisioterapeuta. Mestre e doutora pelo Programa de Pós-Graduação em Saúde e Desenvolvimento da Região Centro Oeste (UFMS). Docente nos Cursos da Área da Saúde da Universidade Católica Dom Bosco (UCDB) E-mail: karla@ucdb.br

${ }^{2}$ Fisioterapeuta. Universidade Católica Dom Bosco (UCDB). E-mail: suellenzama@gmail.com

${ }^{3}$ Enfermeira. Mestre pelo Programa de Pós-Graduação em Saúde e Desenvolvimento da Região Centro Oeste (UFMS). Doutoranda pelo Programa de Biotecnologia da UCDB. Docente do curso de Enfermagem da Universidade Católica Dom Bosco (UCDB). E-mail: debienfermagem@hotmail.com

${ }^{4}$ Médico Pediatra. Prof. Dr. Orientador no Programa de Pós-graduação em Saúde e Desenvolvimento da Região Centro Oeste - Universidade Federal de Mato Grosso do Sul (UFMS).E-mail: dbpalhares@hotmail.com 
Resumo: Este estudo objetivou identificar o conhecimento de parturientes sobre benefícios, práticas facilitadoras e complicadoras do aleitamento materno exclusivo. Foram entrevistadas 30 parturientes antes e após 15 dias do parto. As participantes reportaram como principal vantagem da prática do aleitamento materno os benefícios para a criança $(83,3 \% ; n=25)$, seguido dos benefícios à saúde da mulher $(40,0 \% ; n=12)$. A alegação de não saber os aspectos facilitadores que poderiam auxiliar na manutenção da amamentação foi mais prevalente na primeira entrevista $(p=0,007)$, e a categoria alimentação saudável foi mais citada na segunda entrevista $(p=0,04)$. A falta de leite foi a principal alegação complicadora da amamentação $(40,0 \%$; $n=12)$. Conclui-se que há necessidade do fortalecimento de políticas que promovam o acompanhamento multidisciplinar à gestante na rede básica, desde o pré-natal até os dois anos de idade da criança e o treinamento da prática da amamentação e ordenha, a fim de evitar o desmame precoce.

Palavras-chave: amamentação; leite humano; saúde materno-infantil.

Abstract: This study aimed to identify the knowledge of parturients about benefits, facilitating and complicating practices of exclusive breastfeeding. Thirty parturients were interviewed before and after 15 days of delivery. Participants reported as the main advantage of breastfeeding practices the benefits to the child $(83.3 \%, n=25)$, followed by women's health benefits $(40.0 \%, n=12)$. The claim of not knowing the facilitating aspects that could ease the maintenance of breastfeeding was more prevalent in the first interview ( $p=0.007)$ and the healthy eating category was more cited in the second interview $(p=0.04)$. Lack of milk was the main complicating factor in breastfeeding $(40.0 \%, n=12)$. It is concluded that there is a need of strengthening policies that promote multidisciplinary follow-up to pregnant women in the basic health system, from prenatal to two years of age, and of training in the practice of breastfeeding and milking in order to avoid early weaning.

Key words: breastfeeding; human milk; maternal and child health.

Resumen: Este estudio tuvo como objetivo identificar el conocimiento de madres sobre los beneficios, lo que facilita y complica la práctica de la lactancia materna exclusiva. Treinta madres fueron entrevistadas antes y después de 15 días de la entrega. Las participantes reportaron los beneficios para el niño como la principal ventaja de la lactancia materna $(83,3 \%, n=25)$, seguido de beneficios para la salud de las mujeres $(40,0 \%, n=12)$. La afirmación de no conocer los factores facilitadores que podrían facilitar el mantenimiento de la lactancia materna fue más prevalente en la primera entrevista $(p=0,007)$ y la categoría de alimentación saludable fue mencionada con más frecuencia en la segunda entrevista $(p=0,04)$. La falta de leche fue la reivindicación principal de la lactancia $(40,0 \%, n=12)$. Así que hay necesidad de reforzar las políticas que promuevan un enfoque multidisciplinario para las mujeres embarazadas en la red de salud, desde el prenatal hasta los dos años de edad de los niños, y la formación práctica de la lactancia materna y ordeño para evitar destete precoz.

Palabras clave: la lactancia materna; la leche humana; salud materno-infantil. 


\section{INTRODUÇÃO}

Tanto para os seres humanos quanto para os demais mamíferos, o leite materno é garantia de sobrevivência e perpetuação da espécie, sendo a mãe e sua cria protegidas durante esse momento tão frágil e, ao mesmo tempo, tão poderoso. Esse poder está na capacidade biológica de transporte dos diversos nutrientes necessário para a sobrevivência do filho, seus fatores imunológicos, além dos laços afetivos, um facilitador de todos os processos de desenvolvimento deste novo ser, seja biológico, emocional ou social.

É internacionalmente aceito que as crianças desde seu nascimento recebem leite de suas mães, por ser um produto espécie-específico, e que esse processo perdure de forma exclusiva até o sexto mês, passando a receber alimentação complementar a partir de então, até os 24 meses de vida. Esse padrão, preconizado pela Organização Mundial de Saúde, é considerado a proposta de saúde pública mais impactante para a sociedade, garantindo saúde, desenvolvimento individual e de toda a sociedade, diminuindo drasticamente a mortalidade infantil, e de forma mais expressiva em países em desenvolvimento.

Apesar da implantação, em 2008, da Rede Amamenta Brasil, fomentando aleitamento materno na rede de Atenção Básica à Saúde, a prática de aleitamento materno exclusivo ainda está aquém do ideal, comprometendo tanto a saúde da mãe como de seu filho. Uma vez que as ações do Sistema Único de Saúde (SUS) são descentralizadas, torna-se necessário identificar quais fatores contribuem para esse desmame precoce nas diferentes localidades do País.

Portanto, neste estudo, foram entrevistadas 30 parturientes internadas em um Hospital Universitário na Cidade de Campo Grande, capital do Estado de Mato Grosso do Sul, com o objetivo de identificar seus conhecimentos sobre os benefícios do aleitamento materno exclusivo e sobre as práticas facilitadoras ou complicadoras desse 
processo. Utilizando material disponibilizado nas Unidades Básicas de Saúde pelo Ministério da Saúde, as parturientes foram orientadas sobre o tema. Posteriormente, após 15 dias, essas mulheres, então nutrizes, foram contatadas por telefone para que se pudesse identificar o atual conhecimento destas sobre o tema proposto e suas atuais práticas facilitadoras e e/ou complicadoras do aleitamento materno exclusivo.

Atualmente, compreender o comportamento da população é considerado uma importante ferramenta para mudança de paradigmas, a partir de tecnologias leves de educação em saúde, com baixo custo e grande abrangência social, podendo atingir diferentes extratos da sociedade em uma ação intersetorial, mas fundamentalmente pautada no comportamento responsável dos profissionais da saúde envolvidos no cuidado integral do cidadão brasileiro.

\section{FUNDAMENTAÇÃO TEÓRICA}

O leite materno é fundamental para a saúde da criança, trazendo benefícios ao lactente devido às suas características nutricionais e imunológicas, além de estimular o relacionamento afetivo entre mãe e filho. Assim, a Organização Mundial da Saúde (OMS) preconiza o aleitamento materno exclusivo (AME) até o sexto mês de vida da criança e, a partir de então, deverá ser complementado com outros alimentos até os dois anos ou mais (SANTOS et al., 2009; NEVES et al., 2011).

O AME é uma questão de saúde pública, garantindo a diminuição nos coeficientes de mortalidade infantil. Países em desenvolvimento devem incorporar essa prática visando a uma internalização cultural, uma vez que manter crianças aleitadas exclusivamente por leite materno promove uma redução de $49 \%$ no risco de diarreia em recém-nascidos em relação às crianças amamentadas com outras fórmulas. 
Portanto fica evidente que os aspectos envolvidos na amamentação possuem um impacto profundo da sociedade (BENER; EHLAYEL; ABDULRAHMAN, 2011; BEZERRA et al., 2012).

Com a intenção de mobilizar os profissionais da área da saúde a favor da amamentação e resgatar a prática do aleitamento materno pelos hospitais e maternidades, em 1994, foi criado no Brasil a iniciativa Hospital Amigo da Criança (HAC) para instituições de saúde públicas e privadas, seguindo a estratégia mundial padronizada pela OMS e Unicef, conhecidas como "dez passos para o sucesso do aleitamento materno" (BRASIL, 2011).

Para fortalecer a abrangência das ações de incentivo à amamentação e redução dos índices de mortalidade materna e infantil, em 18 de novembro de 2008, foi instituída, no Sistema Único de Saúde (SUS), a Portaria n. 2.799, que dispõe sobre a Rede Amamenta Brasil, uma estratégia que visa à promoção, proteção e ao apoio do aleitamento materno na rede de Atenção Básica à Saúde. Contudo, apesar dos profissionais da saúde e programas estimularem essa prática, a suspensão precoce da amamentação ainda continua a ocorrer de forma expressiva (CALDEIRA et al., 2007).

O leite materno proporciona inúmeros benefícios para a mãe, dentre estes se destaca a permanência da amenorreia materna pós-parto, enquanto amamenta, principalmente nos 03 primeiros meses, possibilitando maior espaçamento entre as gestações. Também há menor risco de morte por artrite reumatoide, diminuição do risco de câncer de mama, retorno precoce ao peso pré-gestacional e o menor sangramento uterino pós-parto (BRASIL, 2011; 2015).

Extrapolando o tema além das questões puramente fisiológicas, denota-se que o ambiente social e o contexto histórico e cultural que a mulher-mãe-nutriz vivencia afetam diretamente o processo de aleitamento materno, refletindo em graves problemas enfrentados, como as alegações de que o leite é fraco, da pequena produção láctea 
ou respaldados no desconforto presente ao início do processo de amamentação (GIUGLIANI, 2004).

Muitos fatores contribuem para que ocorra o desmame antes do tempo previsto, porém a ausência de conhecimento sobre aleitamento materno pelas gestantes e nutrizes tem demonstrado ser uma das principais causas ao abandono dessa prática, expondo a criança a riscos de desnutrição, de infecção, comprometendo seu crescimento e desenvolvimento (PASSARIN; SANTOS, 2009; ALVES, 2010).

Este estudo teve como objetivo identificar o conhecimento de parturientes sobre os benefícios AME e sobre quais são suas práticas facilitadoras ou complicadoras desse processo.

\section{MÉTODO}

Estudo descritivo transversal realizado no Núcleo Hospital Universitário Maria Aparecida Pedrossian (NHU) - Universidade Federal de Mato Grosso do Sul, Campo Grande, MS, nos meses de janeiro a fevereiro de 2014, após autorização do hospital e aprovação do Comitê de Ética em Pesquisa com Seres Humanos CEP/UCDB (CAAE 24847713.3.0000.5162).

O grupo amostral não probabilístico foi composto por 33 parturientes, definido de acordo com o fluxo do hospital, desde que apresentassem bom estado geral, independente da idade, não se incluindo aquelas em trabalho de parto avançado, assim como muIheres com transtorno mental referido e indígenas. Três parturientes foram excluídas por não contemplarem a segunda fase da pesquisa, resultando em 30 parturientes. O estudo iniciou-se após as participantes ou seus responsáveis legais assinarem o Termo de Consentimento Livre e Esclarecido (TCLE) e, no caso de gestante menor de 18 anos, também a assinatura do termo de assentimento. 
A pesquisa foi dividida em dois momentos: No primeiro momento, a coleta de dados foi realizada pessoalmente no hospital, buscando as ideias explanadas pelas próprias parturientes para que expusessem seus conhecimentos acerca do aleitamento materno, guiadas por um questionário estruturado. Com a autorização prévia da participante, a entrevista foi gravada para transcrição das respostas abertas e posterior análise e categorização. Após a entrevista, foi distribuído um material do Ministério da Saúde com orientações acerca do aleitamento materno e doação de leite humano, sendo estas de caráter motivacional e de promoção à adesão a tais práticas.

No segundo momento, após 15 dias ao primeiro encontro, ou seja, no período em que elas já estavam amamentando, a coleta de dados foi realizada via telefonema, com intuito de identificar como se procedeu a adesão ou não da nutriz às práticas de aleitamento materno.

Os dados nominais e categóricos foram apresentados em distribuição de frequência absoluta e relativa, enquanto as variáveis quantitativas, por meio de média e desvio padrão da média. Aplicouse o teste de Exato de Fisher para analisar associação entre variáveis, considerando significativas associações cujo valor de p for menor 0,05, sendo utilizado o Software estatístico Graph pad 3.06.

\section{RESULTADOS E DISCUSSÃO}

Diversos benefícios da prática da amamentação foram descritos por $96,7 \%$ ( $n=29)$ das parturientes no primeiro momento da entrevista. A análise das respostas apontou que apenas uma parturiente não soube relatar nenhum benefício da prática do aleitamento materno e que o conhecimento da grande maioria delas está voltado aos benefícios que o aleitamento materno proporciona principalmente à saúde da criança $(83,3 \%, n=25)$, com uma valorização da ação nutricional 
$(63,3 \%, n=19)$, imunológica do leite materno $(10,0 \%, n=03)$, corroborando com respostas de parturientes participantes nos estudos de Passarin e Santos (2009), A capacidade de promover dentes mais fortes foi citada por 10,0\% ( $n=03$ ) delas.

A maior valorização dos aspectos nutricionais em si e menor enfoque nas questões imunológicas é parcialmente correta. Inicialmente há produção de colostro, com concentrações de proteínas do soro, fatores de crescimento e proteínas de ação imunológica mais elevadas do que no leite maduro, apresentando menores concentrações de lactose, lipídios e vitaminas do complexo B. Considerando o fator nutricional do leite maduro, apenas após o 150 dia ao parto este se torna completo para o lactente, o chamado leite maduro, apresentando substâncias como água, proteína (em especial a caseína do LH), gordura e vitaminas adequadas para o desenvolvimento saudável da criança (VITOLO, 2008).

O leite materno possui anticorpos específicos às doenças a que as mães tenham sido expostas, protegendo a criança de contraí-las. Em Gana, foi realizado um estudo com dados de 11.316 crianças que foram amamentadas logo após o parto e sobreviveram ao segundo dia após o nascimento, apoiando os aspectos imunológicos do LH, concluindo que a amamentação precoce pode promover a redução dos índices de mortalidade neonatal (EDMOND et al., 2006).

O desenvolvimento neuropsicomotor da criança está relacionado a fatores intrínsecos e extrínsecos e, dentre os nutrientes necessários para um desenvolvimento típico, tem-se a presença de ácidos graxos poliinsaturados de cadeia longa (DHA, EPA e ARA), considerados essenciais para o desenvolvimento do cérebro e da retina. Como o organismo no bebê não sintetiza esses ácidos graxos, eles devem ser essencialmente disponibilizados pela mãe desde a vida intrauterina e após o nascimento pelo leite materno, visto que fórmulas lácteas comerciais são isentas destes lipídeos (PÉREZ-ESCAMILLA; MARTINEZ; SEGURA-PÉREZ, 2016). 
A afetividade foi destacada por 23,3\% ( $n=07)$ das entrevistadas, o que se justifica no reconhecimento internacional de que a amamentação é a primeira e maior estratégia instintiva de vínculo do Ser humano, de afeto, proteção e nutrição para a criança. Foi surpreendente, apenas 6,7\% (n=02) das mulheres destacarem o fator socioeconômico como uma vantagem de aleitamento materno, potencializada pela sua praticidade, por ser considerada a prática mais barata, economicamente viável e de maior efetividade para redução da morbimortalidade infantil (BRASIL, 2015).

Como vantagens da amamentação para as nutrizes, sobressaíram relatos de que o aleitamento materno proporciona emagrecimento eficaz e recuperação mais rápida no pós-parto. O benefício materno relatado por $20 \%$ ( $n=06$ ) das entrevistadas relacionado a uma aceleração ao retorno do peso pré-gestacional mais rápido com a amamentação está correto, baseado no alto gasto energético despendido na lactação, uma vez que para se produzir 1 litro de leite são necessários 900 kcal da nutriz. Sendo assim, torna-se necessário um acréscimo de 500 kcal na ingestão alimentar dessas mulheres, distribuídos entre carboidratos, proteínas e lipídios. Com isso, verifica-se que há um déficit de 400 kcal que pode gerar uma perda de peso (VITOLO, 2008).

Identificar que as parturientes deste estudo relacionam tais benefícios à prática da amamentação é muito positivo, pois esse conhecimento pode fortalecer a manutenção do aleitamento materno exclusivo. A obtenção desse conhecimento pode estar associada ao fato de ter predominado o ensino médio como nível de escolaridade entre as entrevistadas 56,7\% ( $n=17)$, enquanto $30,0 \%(n=9)$ cursaram o ensino fundamental e apenas $13,3 \%(n=04)$ o ensino superior. Estudos nacionais e internacionais têm evidenciado que o conhecimento das mães sobre as vantagens e práticas do aleitamento materno melhora com o aumento no nível de escolaridade (WILHELM et al., 2008; ALVES, 2010; CHAUDHARY; SHAH; RAJA, 2011; MALLIK et al., 2013). 
O benefício à saúde materna citado por 13,3\% (n=04) das parturientes referente à recuperação mais rápida no pós-parto tem respaldo cientifico pelo fato de a amamentação na primeira hora após o parto reduzir o risco de hemorragia pós-parto, devido à ação do hormônio ocitocina liberado pela hipófise, que auxilia o processo de contração uterina acelerando o retorno do útero ao tamanho normal (VITOLO, 2008). A amamentação como fator protetor ao câncer de mama foi relatada por $6,7 \%(n=02)$ das entrevistadas, em consonância com a literatura (BRASIL, 2015).

As respostas associadas às vantagens da amamentação foram agrupadas em categorias e estão detalhadas na tabela 1.

Tabela 1 - Distribuição do conhecimento apresentado pelas parturientes relacionado às vantagens de amamentar. Campo Grande, 2014 ( $n=30)$

\begin{tabular}{lrc}
\hline Vantagens do Aleitamento materno & $\mathbf{n}$ & $\mathbf{\%}$ \\
\hline Saúde do Bebê & 25 & 83,3 \\
Saúde da Mulher & 12 & 40,0 \\
Prática Saudável & 9 & 30,0 \\
Afetividade & 8 & 26,7 \\
Fator socioeconômico & 3 & 10,0 \\
Facilita a produção de leite & 2 & 6,7 \\
Não sabe & 1 & 3,3 \\
\hline
\end{tabular}

Ao questionar as parturientes sobre as desvantagens da prática da amamentação, 70\% ( $n=21)$ delas as desconheciam e 30\% (n=9) afirmaram não ter desvantagem. Apesar disto, quando interrogadas no primeiro momento da entrevista sobre os possíveis fatores complicadores para prática de amamentação, o aspecto mais citado foi a má alimentação materna (20\%; $n=06), 36,7 \%(n=11)$ relataram problemas com as mamas, como dor, rachaduras, mamilo invertido ou 
o leite "empedrar", além de 13,3\% (n=04) mencionaram problemas emocionais.

Após 15 dias ao parto, 23,3\% ( $n=07)$ das nutrizes declararam ter enfrentado dificuldades para amamentar, e algumas das dificuldades citadas anteriormente foram reafirmadas, como os problemas com as mamas (mamilo sensível, mamilo não formado, uma mama mais cheia que a outra) mantiveram percentuais iguais nos dois momentos da entrevista $(16,7 \%, \mathrm{n}=05)$.

As orientações quanto a uma pega adequada da boca do bebê ao seio materno, associadas às técnicas de posicionamento da mãe e manutenção da mama durante os intervalos das mamadas com utilização do próprio leite como cicatrizante são preconizados pelo Ministério da Saúde para evitar lesões na mama e constam como informações fundamentais a serem dadas desde o pré-natal, permanecendo posteriormente como medida de apoio à nutriz e fomento ao aleitamento materno exclusivo até os seis primeiros meses de vida (BRASIL, 2011).

Apesar de que no primeiro momento da entrevista apenas 6,7\% $(n=02)$ das parturientes justificaram a falta de leite como um fator complicador para prática de amamentação, no segundo momento, a falta de leite foi descrita por 40,0\% (n=12) das agora nutrizes, estando esse fator complicador estatisticamente associado ao segundo momento da entrevista, ou seja, durante o processo de lactação $(p=0,005)$.

Corroborando com a literatura, as alegações ao desmame precoce são comuns, como "meu leite é pouco", "meu leite secou" e "meu leite é fraco" (MARQUES; COTTA; PRIORE, 2011). Todavia, geralmente a partir do segundo ao terceiro dia após o parto, a nutriz começa a produzir leite em excesso (VITOLO, 2008).

Para Gonçalves e Bonilha (2005), a crença do leite fraco está fundamentada na comparação do leite humano com o leite de vaca, em que mães acreditam que o leite humano, por ter aparência de 
ser mais aguado, seja nutricionalmente inferior ao leite de vaca, na cultura popular considerado "forte". Já o mito do leite insuficiente é influenciado pelo aspecto psicológico. Fisiologicamente, todas as nutrizes produzem leite em quantidade e qualidade suficiente para seu lactente, desde que se sintam seguras e capazes de desenvolver tal prática (VITOLO, 2008).

Dentre as entrevistadas com dificuldades para amamentar, $16,7 \%$ ( $n=05$ ) já haviam oferecido outro tipo de leite para o lactente, interrompendo o aleitamento materno exclusivo. É importante destacar que usualmente mitos e crenças interferem diretamente na adesão ao aleitamento materno exclusivo, pois o ato de amamentar está fortemente associado a aspectos socioculturais, provocando vários sentimentos na nutriz, como a ansiedade, insegurança, sentimento de culpa e de incapacidade de amamentar, favorecendo o desmame precoce (MARQUES; COTTA; PRIORE, 2011).

O desmame precoce é definido como o período em que há a interrupção do aleitamento materno exclusivo de um lactente através da introdução de qualquer tipo de alimento diferente ao leite materno, quando o principal motivo ao abandono precoce da prática da amamentação associa-se à falta de conhecimento sobre o aleitamento materno entre mães-nutrizes (PASSARIN; SANTOS, 2009; ALVES, 2010).

Comprovando essa influência, averiguou-se na segunda entrevista que, após 15 dias ao parto, 23,3\% das mães já estavam oferecendo outro tipo de leite como complemento, alegando enfrentar dificuldades para amamentar como a diminuição na produção do leite materno, corroborando com estudos que apontam que as queixas relacionadas à interrupção da prática da amamentação pelas nutrizes incluem o leite ter secado, além da recusa do recém-nascido, alegações de que os seios caem com a lactação, que amamentar causa fissuras e a falta de estímulo e auxílio por parte dos profissionais da saúde durante a gestação (MARQUES; COTTA; PRIORE, 2011; BRASIL, 2015). 
Em contrapartida, fisiologicamente, o processo de liberação do leite está vinculado ao controle dos hormônios de origem hipofisária ocorrendo a sua liberação através de estímulos externos, como o contato com o bebê e estímulos emocionais. Sentimentos negativos proporcionados pelo fracasso da amamentação podem promover retenção láctea (VITOLO, 2008).

Foi identificado durante a primeira entrevista que 73,3\% ( $n=22)$ das parturientes haviam realizado mais de 08 consultas pré-natais, contudo 46,7\% ( $n=14$ ) delas não souberam descrever nesse primeiro momento quais os seus conhecimentos sobre os aspectos facilitadores do processo de amamentação. Contudo, após 15 dias do parto, apenas $10 \%(n=3)$ ainda não tinham tal conhecimento, sendo muito significativa a associação entre o segundo momento da entrevista após as orientações das pesquisadoras, somada à vivência do processo de aleitamento e conhecimento da importância desta prática $(p=0,003)$.

Os valores identificados como facilitadores ou complicadores para a manutenção do aleitamento materno nos dois momentos de entrevista estão detalhados na tabela 2. 
Tabela 2- Distribuição dos valores relativos à percepção das participantes aos componentes facilitadores ou complicadores para a manutenção do aleitamento materno durante a primeira entrevista e após 15 dias ao parto. Campo Grande, 2014. (n=30)

\begin{tabular}{|c|c|c|c|c|c|}
\hline \multirow[t]{2}{*}{ Percepções das participantes } & \multicolumn{2}{|c|}{$\begin{array}{c}\text { Primeira } \\
\text { Entrevista }\end{array}$} & \multicolumn{2}{|c|}{$\begin{array}{c}15 \text { dias após } \\
\text { o Parto }\end{array}$} & \multirow{2}{*}{$\begin{array}{l}\text { Valor } \\
\text { de } p\end{array}$} \\
\hline & $\mathbf{n}$ & $\%$ & $\mathbf{n}$ & $\%$ & \\
\hline \multicolumn{6}{|c|}{ Facilitadores para a manutenção do aleitamento materno } \\
\hline Alimentação Saudável & 7 & 23,3 & 16 & 53,3 & $0,03 *$ \\
\hline Ingestão de Líquidos & 5 & 16,7 & 8 & 26,7 & 0,53 \\
\hline Excesso de Leite & 2 & 6,7 & 5 & 16,7 & 0,42 \\
\hline Orientação de como Amamentar & 5 & 16,7 & 1 & 3,3 & 0,19 \\
\hline Boa Saúde Emocional & 3 & 43,3 & 1 & 3,3 & 0,61 \\
\hline Não Sabe & 14 & 46,7 & 3 & 10,0 & $0,003^{*}$ \\
\hline \multicolumn{6}{|c|}{ Complicadores para a manutenção do aleitamento materno } \\
\hline Má alimentação & 6 & 20,0 & - & - & - \\
\hline Problemas com as mamas & 11 & 36,7 & 5 & 16,7 & 0,14 \\
\hline Problemas emocionais & 4 & 13,3 & - & - & - \\
\hline Falta de leite & 2 & 6,7 & 12 & 40 & $0,005^{*}$ \\
\hline
\end{tabular}

Nota: *Significativa associação entre os momentos- Teste Exato de Fisher

Foi possível observar que o número de consultas de pré-natal não foi condição única para o esclarecimento das parturientes sobre o assunto proposto, trazendo o questionamento sobre o equilíbrio entre qualidade e quantidade de informações. O desenvolvimento de programas de treinamento durante o período pré-natal e atividades de educação em saúde sobre o aleitamento materno são cruciais para melhorar o conhecimento, a iniciação e manutenção das práticas do aleitamento materno, garantindo a conservação da saúde da futura geração (CHAUDHARY; SHAH; RAJA, 2011; MALLIK et al., 2013).

Desconheciam sobre a existência de Bancos de Leite Humano (BLH) e de seu papel facilitador no processo de amamentação 53,3\% 
( $n=16)$ das entrevistadas. Conceição et al. (2013) relataram situação semelhante ao entrevistar nutrizes que não haviam recebido orientações no pré-natal a respeito do manejo clinico nas complicações da mama, nem do papel do banco de leite humano (BLH) no processo de amamentação e fomento a doação de leite materno.

O BLH é o local de referência para atendimento às nutrizes que estão com dificuldades na amamentação, principalmente em relação ao ingurgitamento das mamas, fissuras ou mastites, e também para auxiliar na pega correta quando esta não acontece de modo facilitado (CONCEIÇÃO et al., 2013). Auxilia no processo de estabelecimento e direcionamento dos programas educativos, na reorientação das práticas seguidas pelos profissionais e serviços de saúde, na prevenção do desmame precoce, no aumento do número de doadoras e no volume de leite humano coletado (ALMEIDA; MAIA; NOVAK, 2004; NEVES et al., 2011).

Entre os aspectos facilitadores, o número de mulheres que não sabia identificar os fatores facilitadores da amamentação foi significativamente maior no primeiro momento da entrevista $(p=0,003)$. Outra categoria identificada que obteve diferença significativa entre os momentos de entrevista foi a alimentação saudável da nutriz para aumentar sua produção láctea, sendo esse critério mais valorizado durante a vivência da amamentação, evidenciado no segundo momento da entrevista $(p=0,04)$. Inicialmente, 6,7\% das mulheres disseram que amamentar facilita a produção de leite, e esse baixo percentual está significativamente menor que o número de nutrizes, que no segundo momento da entrevista alegaram falta de leite como uma dificuldade para amamentar. Diante dessa problemática, reconhece-se a necessidade de suporte adequado e contínuo às mães-nutrizes, pois geralmente estas desejam e iniciam a amamentação, como se observa neste estudo. No entanto esse ato inicial, muitas vezes, não é suficiente para a manutenção do aleitamento devido a diversas situações às 
quais ela está sujeita, como o ingurgitamento mamário, problemas com as mamas e os mitos e crenças, que induzem a introdução de outros alimentos levando ao desmame (CALDEIRA et al., 2007).

Considerando os principais facilitadores para a manutenção da amamentação relatados durante o primeiro momento da pesquisa, destaca-se o excesso de leite e alimentação saudável, com ênfase na ingestão de derivados do milho, como a canjica. Após 15 dias ao parto, os facilitadores citados durante a primeira entrevista foram reafirmados como adjuvantes no processo do aleitamento materno, todavia, apesar de todas as mães terem aderido à prática da amamentação, algumas já haviam abandonado o aleitamento materno exclusivo.

O milho é um cereal e o principal substrato da canjica. Frequentemente é alegada entre as nutrizes como alimento que garante a produção de leite humano. Os cereais são divididos em três grupos que o compõem: o pericarpo, no qual se encontra a fibra alimentar; germe, porção onde se localiza a gordura e, por fim, endosperma que é rica em carboidrato. Um estudo avaliou a composição da canjica, concluindo que ela não possuiu muito pericarpo e germe, logo prevalecendo o endosperma em sua constituição, caracterizando assim um alimento energético e com o acréscimo do leite em sua preparação proporciona um aporte hídrico para a nutriz (CALLERGARO et al., 2005).

Pode-se considerar que este é um mito até certo ponto benéfico à mãe-nutriz, mas, quando há adição excessiva de açúcar e outros alimentos de valores calóricos, torna-se prejudicial àquelas que possuem problemas crônicos como obesidade ou diabetes.

A maioria das participantes $(63,3 \%, n=19)$ se declarou em união estável como estado civil, apresentando variação de idade entre 15 e

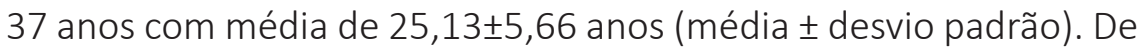
acordo Wilhelm et al. (2008), esses fatores somados a uma maior escolaridade (neste, estudo ensino médio foi predominante) configuram 
uma população com estrutura socioeconômica e emocional capaz de maior autoeficiência em relação ao aleitamento materno.

Outro fator determinante para o aleitamento materno exclusivo esteve relacionado ao fato de os partos terem sido predominantemente a termo, com tempo de gestação 37 a 41 semanas, com média

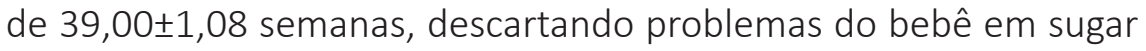
a mama. As crianças que não puderam receber alta com a mãe em decorrências de complicações peri ou pós-natais foram excluídas deste estudo. Outra questão que deveria facilitar o processo de amamentação é a maior prevalência de multíparas $(53,3 \%, n=16)$, cuja experiência prévia poderia minimizar as fantasias, medos e anseios em relação a esse processo de amamentação (GREGORY et al., 2008; MCCARTER-SPAULDING; GORE, 2009).

Até dezembro de 2010, foram realizadas quatro oficinas de capacitação da Rede Amamenta Brasil no estado de Mato Grosso do Sul, duas em Campo Grande, uma em Corumbá e uma em Dourados, com a participação de 3.573 profissionais da saúde, 186 Unidades Básicas de Saúde (UBS) e formação de 133 tutores (BRASIL, 2011). A partir dessa informação, pode-se sugerir a presença de falhas no processo de treinamento dos profissionais de Campo Grande, MS, já que 16,7\% das puérperas haviam interrompido o AME antes dos 15 dias de vida do lactante.

Brandão, Venancio e Giugliani (2015) desenvolveram um estudo no sul do Brasil e identificaram que um quarto dos profissionais atuantes em Unidades Básicas de Saúde (UBS) que estavam aderindo à Rede Amamenta Brasil no referido serviço, desconheciam a existência de fluxogramas de atendimento à dupla mãe-bebê durante o período de amamentação e não identificaram diferença entre as crianças assistidas nas unidades que não utilizavam protocolos de manejo do AM e as das unidades que aderiram ao programa Rede Amamenta Brasil. 
O acesso a informações sobre práticas saudáveis para a nutriz e o bebê, ressaltando a importância do aleitamento materno exclusivo durante os primeiros seis meses de vida, contribui para o êxito da amamentação. O incentivo ao aleitamento materno representa uma das principais ações em saúde, sendo os profissionais da saúde os atores responsáveis por sua abrangência e sucesso (BRASIL, 2011).

Porém,

[...] a implementação das ações de proteção e promoção do aleitamento materno e da adequada alimentação complementar depende de esforços coletivos intersetoriais e constitui enorme desafio para o sistema de saúde, numa perspectiva de abordagem integral e humanizada. (BRASIL, 2015, p. 7).

Destacam-se algumas limitações do estudo relacionadas a um número restrito de parturientes internadas no período proposto, devido à reorientação do sistema de vagas do município, com uma em média de duas a três entrevistas por dia. No segundo momento de entrevista, houve dificuldades durante as ligações, pois as mães alegavam que estavam ocupadas com o lactente, havendo a necessidade de realizar várias tentativas durante o dia até a assertiva das 30 participantes.

\section{CONCLUSÕES}

O conhecimento sobre aspectos importantes da amamentação, como os benefícios proporcionados à saúde da nutriz e da criança e associação entre hábitos saudáveis como facilitadores desse processo, não foi suficiente para que todas mantivessem o aleitamento materno exclusivo, sugerindo que, apesar de ser necessária, a obtenção do conhecimento não é suficiente para garantir a adesão da mulher-nutriz à prática da amamentação. 
Apesar das ações para a promoção da adesão ao aleitamento materno, os fatores socioculturais e psicossociais continuam proporcionando o desmame precoce.

Ressalta-se a necessidade do fortalecimento de políticas que promovam o acompanhamento multidisciplinar à gestante na rede básica de atenção à saúde, fomentando o acompanhamento adequado e contínuo, desde o pré-natal até os 6 meses de idade da criança, período do aleitamento materno exclusivo, em que a equipe se responsabiliza pelo cuidado em saúde, trabalhando não apenas atividades de educação em saúde relacionadas ao tema, mas enfatizando o treinamento da prática da amamentação e ordenha, já que as dúvidas sobre a qualidade do leite e problemas com as mamas enfrentados pelas nutrizes são os principais motivos ao desmame precoce.

\section{REFERÊNCIAS}

ALMEIDA, J. A. G; MAIA, P. R. S; NOVAK, F. R. Os bancos de leite humano como suporte para a redução da mortalidade infantil: a experiência brasileira. In: CONGRESSO URUGUAYO DE LACTANCIA MATERNA, 2., Montevideo, Uruguay. Anais... Montevideo: Sociedad Uruguaya de Pediatria, 2004. Disponível em: <http://www.bvsam.cict.fiocruz.br/evcientif/2culm/2culm.htm>. Acesso em: 27 dez. 2013.

ALVES E.A. Fatores determinantes do desmame precoce: um estudo de revisão bibliográfica. 2010. Monografia (Especialização em Atenção Básica em Saúde da Família)- Universidade Federal de Minas Gerais, Belo Horizonte, 2010.

BENER, A.; EHLAYEL, M. S.; ABDULRAHMAN, H. M. Exclusive breast feeding and prevention of diarrheal diseases. A study in Qatar. Revista Brasileira de Saúde Materno Infantil, Recife, v. 11, n. 1, p. 83-87, jan./mar. 2011.

BEZERRA, V. L. V. A.; NISIYAMA, A. L.; JORGE, A. L.; CARDOSO, R. M.; SILVA, E. F.; TRISTÃO, R. M. Aleitamento materno exclusivo e fatores associados a sua interrupção precoce: estudo comparativo entre 1999 e 2008. Revista Paulista de Pediatria, São Paulo, v. 30, n. 2, p. 173-9, jun. 2012. 
BRANDÃO, D. S.; VENANCIO, I. S.; GIUGLIANI, E. R. J. Association between the Brazilian Breastfeeding Network implementation and breastfeeding indicators. Jornal de Pediatria, Rio de Janeiro, v. 91, n. 2, p. 143-51, mar./abr. 2015.

BRASIL. Ministério da Saúde. Secretaria de Atenção à Saúde. Departamento de Atenção Básica. Saúde da criança: aleitamento materno e alimentação complementar. 2. ed. Brasília: Ministério da Saúde, 2015. 184 p.: il. (Cadernos de Atenção Básica, n. 23).

. Ministério da Saúde. Secretaria de Atenção à Saúde. Área Técnica de Saúde da Criança e Aleitamento Materno. Rede Amamenta Brasil: os primeiros passos (2007-2010). Brasília: Ministério da Saúde, 2011.

CALDEIRA, A. P.; AGUIAR, G. N. D.; MAGALHÃES, W. A. C.; FAGUNDES, G. C. Conhecimentos e práticas de promoção do aleitamento materno em Equipes de Saúde da Família em Montes Claros, Minas Gerais, Brasil. Cadernos de Saúde Pública, Rio de Janeiro, v. 23, n. 8, p. 1965-70, ago. 2007.

CALLERGARO, M. G. K.; DUTRA, C. B.; HUBER, L. S.; BECKER, L. V.; ROSA, C. S.; KUBOTA, E. H.; HECKTHEUR, L. H. Determinação de fibra alimentar insolúvel, solúvel e total de produtos derivados do milho. Ciência e Tecnologia de Alimentos, Campinas, SP, v. 25, n. 2, p. 271-4, abr./jun. 2005.

CHAUDHARY, R. N.; SHAH, T.; RAJA, S. Knowledge and practice of mothers regarding breast feeding: a hospital based study. Health Renaissance, v. 9, n. 3, p. 194-200, 2011.

CONCEIÇÃO, C. S.; ALVES, V. H.; SILVA, L. R.; MARTINS, C. A.; MATTOS, D. V.; RODRIGUES, D. P. Qualidade assistencial do banco de leite humano: percepção de usuárias. Revista de Enfermagem UFPE on line, Recife, v. 7, n. 5, p. 1271-8, maio 2013.

EDMOND, K. M.; ZANDOH, C.; QUIGLEY, M. A.; AMENGA-ETEGO, S.; OWUSU-AGVEI, S.; KIRKWOOD, B. R. Delayed breastfeeding initiation in creasesrisk of neonatal mortality. Pediatrics, v. 117, n. 3, p. 380-6, mar. 2006.

GIUGLIANI, E. R. J. Problemas comuns na lactação e seu manejo. Jornal de Pediatria, Rio de Janeiro, v. 80, n. 5 (supl.), p. S147-S154, nov. 2004.

GONÇALVES, A. C.; BONILHA, A. L. L. Crenças e práticas da nutriz e seus familiares no aleitamento materno. Revista Gaúcha de Enfermagem, Porto Alegre, RS, v. 26, n. 3, p. 333-44, dez. 2005. 
GREGORY, A.; PENROSE, K.; MORRISON, C.; DENNIS, C. L.; MACARTHUR, C. Psychometric properties of the Breastfeeding Self-Efficacy Scale-Short Form in an ethnically diverse U.K. sample. Public Health Nursing, v. 25, n. 3, p. 278-84, maio/jun. 2008.

MALLIK, S.; DASGUPTA, U.; SUBHRAJYOTI, N.; SENGUPTA, D.; CHOUDHURY, K.; BHATTACHARYA, K. S. Knowledge of breast feeding and timely initiation of it amongst post natal mothers: An experience from a baby friendly teaching hospital of a metropolitan city. IOSR Journal of Dental and Medical Sciences, v. 4, n. 1, p. 25-30, 2013.

MARQUES, E. S.; COTTA, R. M. M.; PRIORE, S. E. Mitos e crenças sobre o aleitamento materno. Ciência \& Saúde Coletiva, Rio de Janeiro, v. 16, n. 5, p. 2461-8, maio 2011.

MCCARTER-SPAULDING, D. E.; GORE, R. Breastfeeding self-efficacy in women of African descent. Journal of Obstetric, Gynecologic \& Neonatal Nursing, v. 38, n. 2, p. 230-43, mar./abr. 2009.

NEVES, L. S.; MATTAR, M. J. G.; SÁ, M. V. M.; GALISA, M. S. Doação de leite humano: dificuldades e fatores limitantes. O Mundo da Saúde, v. 35, n. 2, p. 156-161, 2011.

PASSARIN, G. L.; SANTOS, J. S. D. Conhecimento do aleitamento materno em puérperas no Hospital Geral: Caxias do Sul. Pediatria, São Paulo, v. 31, n. 3, p. 152-60, set. 2009.

PÉREZ-ESCAMILLA, R.; MARTINEZ, J. L.; SEGURA-PÉREZ, S. Impact of the Babyfriendly Hospital Initiative on breastfeeding and child health outcomes: a systematic review. Maternal \& Child Nutrition, v. 12, n. 3, p. 402-17, jul. 2016.

SANTOS D. T. D.; VANNUCHI M. T. O.; OLIVEIRA M. B.; DALMAS J. C. Perfil das doadoras de leite do banco de leite humano de um hospital universitário. Acta Scientiarum. Health Science, Maringá, PR, v. 31, n. 1, p. 15-21, 2009.

VITOLO, M. R. Nutrição da gestação ao envelhecimento. Rio de Janeiro: Rubio, 2008.

WILHELM, S. L.; RODEHORST, T. K.; STEPANS, M. B.; HERTZOG, M.; BERENS, $C$. Influence of intention and self-efficacy levels on duration of breastfeeding for midwest rural mothers. Applied Nursing Research, v. 21, n. 3, p. 123-30, ago. 2008. 
